\title{
A NOTE ON GPIS AND THEIR COEFFICIENTS
}

\author{
CHARLES LANSKI
}

\begin{abstract}
If $R$ is a prime ring satisfying a GPI, then $R$ satisfies a multilinear GPI having all its coefficients in $R$. Also, all $R$ bimodules in the Martindale quotient ring of $R$ satisfy the same multilinear GPIs.
\end{abstract}

The purpose of this note is to observe two facts about generalized polynomial identities for prime rings. These are of some general interest and do not seem to have been noticed before. We first review some of the necessary background. Let $R$ denote a prime ring, $C$ its extended centroid, and $Q$ its Martindale quotient ring (see [2] for details). One can view $Q$ as equivalence classes of left $R$-module homomorphisms from ideals of $R$ to $R$, so for each $q \in Q$, there is a nonzero ideal $I_{q}$ of $R$ with $\left(I_{q}\right) q \subset R$, and $q=0$ if $(J) q=0$ for any nonzero ideal $J$ of $R$. It follows that $R$ embeds in $Q$ as right multiplications, and that $C$, which is the center of $Q$, can be characterized as the $R$ bimodule maps from ideals of $R$ to $R$. It is straightforward to check that $Q$ is a prime ring, and that $C$ is the extended centroid of $Q$. Let $F=Q_{C}^{*} C\{x\}$ be the free product over $C$ of $Q$ and the free algebra over $C$ in the set $X=\left\{x_{1}, x_{2}, \ldots\right\}$. An element of $F$ can be represented as a finite sum of monomials, each having the form $q_{0} y_{1} q_{1} \cdots y_{n} q_{n}$ where $\left\{q_{0}, \ldots, q_{n}\right\} \subset Q$ are the coefficients and $\left\{y_{i}\right\} \subset X$. The coefficients of $f \in F$ are all the elements of $Q$ appearing as coefficients in the monomials appearing in the given representation of $f$.

Let $T$ be any nonzero $R$-subbimodule of $Q$. We say that $T$ satisfies a $Q$ generalized polynomial identity ( $Q$-GPI) if for some $f\left(x_{1}, \ldots, x_{n}\right) \in F-\{0\}$, $f\left(t_{1}, \ldots, t_{n}\right)=0$ for all substitutions of $t_{i} \in T$ for $x_{i}$. This notion generalizes the usual situation when $R$ satisfies a GPI, which in our terms means a $Q$-GPI with coefficients in $R C+C$, the central closure of $R$. We shall show that the set of multilinear and homogeneous $Q$-GPIs is the same for any $R$-subbimodule of $Q$, and that if $R$ satisfies a $Q$-GPI, then it satisfies one having all its coefficients in $R$. Before proceeding, we observe that if $I$ is any nonzero ideal of $R$ and $T$ is any nonzero $R$-subbimodule of $Q$, then $T \cap I$ contains a nonzero ideal of $R$, namely $L_{y} y I$ for $y \in T$.

THEOREM 1. Let $T$ be a nonzero $R$-subbimodule of $Q$. If $f \in F$ is a multilinear and homogeneous $Q$-GPI for $T$, then $f$ is a $Q$-GPI for $Q$.

ProOF. Write $f=f\left(x_{1}, \ldots, x_{n}\right)$ and proceed by induction on $n$. The case $n=1$, that is $f=\sum_{i=1}^{m} a_{i} x b_{i}$, uses a modification of $[1$, Lemma 1.3.2, p. 22] to show that $f=0$ in $F$. If $m=1$, use the observation above to find an ideal $J$ of $R$ with $J \subset T \cap I_{b}$, so that $0=I_{a} f(J)=\left(I_{a} a\right) J b$. Since $R$ is prime, either

Received by the editors September 5, 1985.

1980 Mathematics Subject Classification. Primary 16A38; Secondary 16A12, 16 A08.

Key words and phrases. Generalized polynomial identity. 
$a=0$ or $b=0$. if $m>1$ we may assume that $\left\{a_{i}\right\}$ is $C$-independent and all $b_{i} \neq 0$, since otherwise we may rewrite $f$ and use induction on $m$ to obtain $f=0$. Choose a nonzero ideal $J \subset R$ with $J \subset T$ and $J b_{i} \subset R$ for all $i$. Suppose $\left\{x_{j}, y_{j}\right\} \subset J$ satisfies $\sum x_{j} b_{1} y_{j}=0$. Then for all $t \in T, 0=\sum_{j} a_{1} t x_{j} b_{1} y_{j}=$ $-\sum_{i=2}^{m} a_{i} t\left(\sum_{j} x_{j} b_{i} y_{j}\right)$, so induction on $m$ shows $\sum x_{j} b_{i} y_{j}=0$ for $i>1$. It follows that $\left(\sum x_{j} b_{1} y_{j}\right) g_{i}=\sum x_{j} b_{i} y_{j}$ is an $R$-bimodule map from $J b_{1} J$ to $R$, so is given by multiplication by $c_{i} \in C$. Therefore, $J\left(b_{i}-b_{1} c_{i}\right) J \doteq 0$, forcing $b_{j}=b_{1} c_{j}$, and enabling us to write $f=\left(a_{1}+\sum a_{i} c_{i}\right) x b_{1}$. We may conclude that $f=0$ from the case $m=1$.

Suppose now that $f\left(x_{1}, \ldots, x_{n}\right)$ is not a $Q$-GPI for $Q$. Choose $\left\{q_{i}\right\} \subset Q$ with $f\left(q_{1}, \ldots, q_{n}\right) \neq 0$. Clearly, $f\left(x_{1}, q_{2}, \ldots, q_{n}\right) \neq 0$ in $F$, so by the case above there is $t \in T$ with $f\left(t, q_{2}, \ldots, q_{n}\right) \neq 0$. In other words, $f\left(t, x_{2}, \ldots, x_{n}\right)$ is not a $Q$-GPI for $Q$. But $f\left(t, x_{2}, \ldots, x_{n}\right)$ is a multilinear and homogeneous $Q$-GPI for $T$ of degree $n-1$, so induction on $n$ gives the contradiction that it is a $Q$-GPI for $Q$, completing the proof of the theorem.

COROLlaRY 1. If $S$ and $T$ are nonzero $R$-subbimodules of $Q$, then $S$ and $T$ satisfy the same multilinear and homogeneous Q-GPIs.

COROLlaRY 2. If $R$ satisfies a multilinear and homogeneous GPI $f$, then $Q$ also satisfies $f$.

THEOREM 2. Let $T$ and $S$ be any nonzero $R$-subbimodules of $Q$. If $T$ satisfies a Q-GPI, then $T$ satisfies some Q-GPI having all its coefficients in $S$.

ProOF. Using Corollary 1 it suffices to prove that $T$ satisfies a multilinear and homogeneous $Q$-GPI with coefficients in $T$. By the usual linearization procedure we may assume that $T$ satisfies a nonzero multilinear and homogeneous $Q$-GPI, so $Q$ satisfies the same identity by Theorem 1. It follows from Martindales's theorem [2, Theorem 3, p. 579] that $Q C=Q$ is a primitive ring containing a minimal right ideal $e Q$, with $e^{2}=e$, and that $e Q e$ is a finite dimensional division algebra over its center $e C$. Let

$$
S_{2 n}\left(x_{1}, \ldots, x_{2 n}\right)=\sum_{g}(-1)^{g} x_{g(1)} \cdots x_{g(2 n)},
$$

where $g$ ranges over all permutations of $\{1, \ldots, 2 n\}$, be a standard identity satisfied by $e Q e$. Choose a nonzero ideal $J$ of $R$ with $J \subset T \cap I_{e}$, and let $y \in J^{2}$ so that $y e=t \in T-\{0\}$. Clearly, $S_{2 n}\left(e x_{1} y e, \ldots, e x_{2 n} y e\right)$ is a multilinear and homogeneous $Q$-GPI for $Q$, so

$$
\begin{aligned}
f\left(x_{1}, \ldots, x_{2 n}\right) & =y S_{2 n}\left(e x_{1} t, \ldots, e x_{2 n} t\right) \\
& =\sum_{g}(-1)^{g} t x_{g(1)} t \cdots t x_{g(2 n)} t
\end{aligned}
$$

is a multilinear and homogeneous $Q$-GPI for $T$ having coefficients in $T$. Note that $f \neq 0$ since its monomials can be taken as part of a basis of $F$.

COROLLARY. If $R$ satisfies a GPI, then $R$ satisfies a GPI having all its coeffcients in $R$. 


\section{REFERENCES}

1. I. N. Herstein, Rings with involution, Univ. of Chicago Press, Chicago, 1976.

2. W. S. Martindale III, Prime rings satisfying a generalized polynomial identity, J. Algebra 12 (1969), 574-584.

Department of MAThematics, UNiversity of SOUTHERN CALIFORNiA, LOS ANGELES, CALIFORNIA 90089-1113 